\title{
Rotating Black Holes in Randall-Sundrum II Braneworlds
}

\author{
William D. Biggs@ and Jorge E. Santos \\ Department of Applied Mathematics and Theoretical Physics, University of Cambridge, Cambridge, CB3 OWA, United Kingdom
}

(Received 17 September 2021; accepted 30 November 2021; published 10 January 2022)

\begin{abstract}
We find rotating black hole solutions in the Randall-Sundrum II (RSII) model, by numerically solving a three-dimensional PDE problem using pseudospectral collocation methods. We compute the area and equatorial innermost stable orbits of these solutions. For large black holes compared with the AdS length scale $\ell$ the black hole exhibits four-dimensional behavior, approaching the Kerr metric on the brane, while for small black holes, the solution tends instead towards a five-dimensional Myers-Perry black hole with a single nonzero rotation parameter aligned with the brane. This departure from exact four-dimensional gravity may lead to different phenomenological predictions for rotating black holes in the RSII model to those in standard four-dimensional general relativity. This Letter provides a stepping stone for studying such modifications.
\end{abstract}

DOI: 10.1103/PhysRevLett.128.021601

Introduction.-For some time there has been great interest in the idea that our Universe is a brane embedded in a higher dimensional space. In most such theories, the extra dimensions are compact and very small, so that fourdimensional physics is reproduced. The five-dimensional RSII braneworld model [1], on the other hand, contains an extra dimension that is noncompact but warped so that the low-energy behavior of gravity on the brane still yields four-dimensional general relativity. The Randall-Sundrum paradigm [2], was first introduced as a mechanism for solving the hierarchy problem: due to the warping factor, the effective mass of an object in the four-dimensional theory is exponentially suppressed compared with its proper five-dimensional mass, leading to a large hierarchy between gravity and the other forces. Whilst matter fields may be restricted to the brane, gravity (at least at higher energies) must be able to propagate through all dimensions. This motivates finding black hole solutions in the RSII model, since these may yield different phenomenological predictions to ordinary four-dimensional general relativity.

Initially there was some debate over whether stable, large black holes on the brane could exist. Because of arguments from the AdS/CFT correspondence [3-5], the low-energy theory on the brane can be described by gravity coupled to a large $N$, strongly coupled CFT [6-8]. Therefore a black hole in the five-dimensional RSII bulk would correspond holographically to a quantum-corrected black hole on the brane [9]. It was thought that the black hole would quickly

Published by the American Physical Society under the terms of the Creative Commons Attribution 4.0 International license. Further distribution of this work must maintain attribution to the author(s) and the published article's title, journal citation, and DOI. Funded by SCOAP ${ }^{3}$. evaporate due to extra radiation arising from the CFT degrees of freedom, meaning any bulk black hole solution would be time dependent. However, it was contended that this argument neglects the strong coupling of the CFT [10]. The debate was settled in Ref. [11], where static, stable black hole solutions on the brane were found for both small and large radii. This solution is closely related to black droplets and funnels [12-23], which are the gravitational duals to the limit where the CFT decouples from gravity.

In order for the RSII model to be phenomenologically viable it must admit not only static black hole solutions, but also rotating ones. In this Letter we present the first fully backreacted, rotating black hole solution in the RSII model, by utilising the method pioneered in Ref. [24]. We were able to find solutions over a two parameter space. One parameter runs over the possible angular velocity of the black hole, and we were able to find solutions close to extremality. The second parameter controls the size of the black hole relative to the five-dimensional AdS length, $\ell$. We found that for large rotating RSII black holes, the induced metric on the brane closely resembles the fourdimensional Kerr black hole, while small rotating RSII black holes exhibit five-dimensional behavior, approaching the five-dimensional Myers-Perry black holes [25] with a single nonzero rotation aligned with the brane.

This transition from four-dimensional to five-dimensional behavior means that finite-sized RSII black holes have slightly different induced geometry on the brane to the usual four-dimensional Kerr black hole, and thus will lead to different phenomenological predictions for fourdimensional observers. Since the black holes in our Universe are expected to be rotating and uncharged, this provides the possibility of testing the RSII model via astrophysical measurements of black holes as well as in future lepton colliders, such as the International Linear 
Collider [26,27] and the Compact Linear Collider [28]. For example, we show that the radius of the innermost stable equatorial circular orbits (ISCOs) predicted by our solutions differ slightly to that of the Kerr black hole of the same rotation.

Constructing rotating RSII black holes.-We seek a solution to the five-dimensional Einstein equation with negative cosmological constant,

$$
R_{a b}+\frac{4}{\ell^{2}} g_{a b}=0,
$$

where $\ell$ is the five-dimensional AdS radius. To motivate our choice of coordinates, we first consider $\mathrm{AdS}_{5}$ in Poincaré coordinates:

$g_{\mathrm{AdS}_{5}}=\frac{\ell^{2}}{z^{2}}\left[-d t^{2}+d z^{2}+d r^{2}+r^{2}\left(d \theta^{2}+\sin ^{2} \theta d \varphi^{2}\right)\right]$,

where the last two terms correspond to the line element of the round two-sphere with $\theta \in(0, \pi), \varphi \sim \varphi+2 \pi$ being the standard polar and azimuthal angles, respectively.

The RSII model with no matter can be thought of as a part of the Poincaré patch of AdS between the Poincaré horizon and a constant $z$ slice (the brane), with a $\mathbb{Z}_{2}$ reflection symmetry across the brane. This motivates taking the transformations,

$$
z=\frac{\Delta(x, y)}{1-y^{2}}, \quad r=\frac{x \sqrt{2-x^{2}}}{1-y^{2}},
$$

where $\Delta(x, y)=1-x^{2}+\beta^{-1}\left(1-y^{2}\right)$. Now the coordinates $x \in(0,1), y \in(0,1)$ parametrize the space between a brane at $x=1$ (which is a surface of constant $z=\beta^{-1}$ ), and the Poincaré horizon at $y=1$, and so are a natural set of coordinates to find solutions on the RSII brane. The metric becomes

$$
\begin{aligned}
g_{\mathrm{AdS}_{5}}= & \frac{\ell^{2}}{\Delta(x, y)^{2}}\left[-h(y)^{2} d t^{2}+\frac{4 y^{2}}{h(y)^{2}} d y^{2}\right. \\
& \left.+\frac{4}{g(x)} d x^{2}+x^{2} g(x) d \Omega_{(2)}^{2}\right],
\end{aligned}
$$

where, here and throughout, $g(x)=2-x^{2}$ and $h(y)=1-y^{2}$. These were the coordinates used by Ref. [11] to find a static black hole on the brane. In our case, we seek to find a rotating black hole on the brane, and hence our solution will be neither static nor spherically symmetric. However, we do assume that the solution will be stationary and axisymmetric (with Killing vector fields $\partial_{t}$ and $\partial_{\phi}$, respectively), and that it will be invariant under the transformation of simultaneously swapping the signs of $t$ and $\phi$. The most general ansatz, with a bifurcate Killing horizon at $y=0$, satisfying these assumptions is

$$
\begin{aligned}
d s^{2}= & \frac{\ell^{2}}{\Delta(x, y)^{2}}\left\{-\frac{h(y)^{2} y^{2} P(y)}{A(y, u)} F_{1} d t^{2}+\frac{4 F_{2}}{P(y) h(y)^{2}} d y^{2}+\frac{4 F_{5}}{g(x)}\left(d x+\frac{F_{8}}{h(y)} d y+F_{9} d u\right)^{2}\right. \\
& \left.+x^{2} g(x)\left[\frac{4 F_{3}}{2-u^{2}}\left(d u+\frac{F_{7}}{x h(y)} d y\right)^{2}+\left(1-u^{2}\right)^{2} S(x, y, u) F_{4}\left(d \phi+y^{2} W(y, u) F_{6} d t\right)^{2}\right]\right\},
\end{aligned}
$$

where the coordinate $u$ measures the inclination from the equatorial plane and, in pure $\mathrm{AdS}_{5}$, is related to the usual polar angle on the $S^{2}$ via $u\left(2-u^{2}\right)^{1 / 2}=\cos \theta$. The unknown functions $F_{i}$ for $i=1, \ldots, 9$ depend on $\{x, y, u\}$, while $A, P, S$, and $W$ are known functions, given in the Supplemental Material [29], depending on a parameter $\alpha$, which controls the horizon angular velocity of the braneworld black hole. The coordinate domain is the cube $\{x, y, u\} \in(0,1)^{3}$.

The DeTurck method: In order to solve the Einstein equation (1) we use the DeTurck trick [24,31,32]. Effectively, we fix the gauge by adding on a term and solve the resulting equation, which is usually called the EinsteinDeTurck equation or the harmonic Einstein equation:

$$
R_{a b}+\frac{4}{\ell^{2}} g_{a b}-\nabla_{(a} \xi_{b)}=0,
$$

where the DeTurck vector is defined by $\xi^{a}=$ $g^{c d}\left[\Gamma_{c d}^{a}(g)-\Gamma_{c d}^{a}(\bar{g})\right]$. Here, $\Gamma_{c d}^{a}(\mathfrak{g})$ is the Christoffel connection associated to a metric $\mathfrak{g}$, and $\bar{g}_{a b}$ is a reference metric which we are free to choose. After adding on this gauge fixing term, demanding our symmetry restrictions and imposing appropriate boundary conditions, the EinsteinDeTurck equation becomes a set of elliptic partial differential equations.

Note, however, that in order for a solution to the Einstein-DeTurck equation to also yield a solution to Einstein's equation, we need $\xi^{a}=0$ on solutions of (6). A solution with nonzero $\xi^{a}$ is called a Ricci soliton. It has been shown in certain cases that Ricci solitons cannot exist [16,33], however, our work does not satisfy the required conditions to make this claim a priori. Instead, we simply solve the Einstein-DeTurck equation and check afterwards that the DeTurck vector is small and tending towards zero in the continuum limit. Since the problem we are solving is elliptic, we know that solutions with nonzero $\xi$ cannot be arbitrarily close to a solution of Einstein's equation. As such we use the norm $\xi^{2}$ to monitor whether we are approaching a true solution of the Einstein equation (1). 
In our case, we choose the reference metric to be defined by the above ansatz with

$$
F_{i}(x, y, u)= \begin{cases}1 & \text { for } 1 \leq i \leq 5 \\ \frac{\alpha}{1+\alpha^{2}} & \text { for } i=6 \\ 0 & \text { for } 7 \leq i \leq 9\end{cases}
$$

Boundary conditions: First, let us consider the fictitious boundaries of our domain. These consist of the equatorial plane at $u=0$, the north pole at $u=1$, the axis of symmetry where the $S^{2}$ shrinks to zero size at $x=0$ and the bifurcating Killing horizon at $y=0$. The boundary conditions at each of these fictitious boundaries, which can be found in the Supplemental Material [29], are fixed by requiring regularity.

At the $y=1$ boundary, we require that the metric approaches the Poincare horizon of $\mathrm{AdS}_{5}$. In order to achieve this, we enforce that the metric is equal to the reference metric at this boundary. Since $A, P, S$, and $W$ are all equal to one at $y=1$, note that this boundary condition means the metric matches (4) as $y \rightarrow 1^{-}$after the identification, $d \varphi=d \phi+\alpha /\left(1+\alpha^{2}\right) d t$.

Finally, we need to consider the boundary conditions on the brane (located at $x=1$ ). In the RSII setup, the two sides of the brane are identified under a $\mathbb{Z}_{2}$ symmetry. Since we are interested in the case where the stress energy tensor on the brane vanishes, the corresponding Israel junction conditions [34] read

$$
0=K_{a b}-K \gamma_{a b}+\frac{3}{\ell} \gamma_{a b},
$$

where $\gamma_{a b}$ is the induced metric on the brane and $K_{a b}=$ $\gamma_{a c} \nabla^{c} n_{b}$ is the extrinsic curvature, with $n_{b}$ being the inward unit normal to the brane. These provide six boundary conditions on the brane. We additionally impose that $\xi_{x}=0$ and that $F_{8}=F_{9}=0$ at $x=1$, making a total of nine boundary conditions, imposed on the nine functions $F_{i}$.

Numerics: We approximate the PDEs by a set of nonlinear algebraic equations defined on a Gauss-Lobatto grid using pseudospectral collocation methods. The resultant algebraic equations are then solved using a standard Newton-Raphson algorithm (see, for instance, Ref. [32] for a comprehensive review of such methods).

Our ansatz depends on two parameters: $\alpha$ and $\beta$. The parameter $\alpha$ controls the rotation of the black hole, with $\alpha=1$ being an extremal black hole and $\alpha=0$ the static braneworld black hole of Ref. [11]. The size of the black hole relative to the AdS length scale $\ell$ is in turn controlled by $\beta$. More specifically, the temperature and angular velocity of the braneworld black hole, measured with respect to inertial coordinates at spatial infinity of the braneworld, is given by $T=(4 \pi \ell \beta)^{-1}\left(1-\alpha^{2}\right) /\left(1+\alpha^{2}\right)$ and $\Omega=\alpha(\ell \beta)^{-1} /\left(1+\alpha^{2}\right)$, respectively. Note that the ratio $\Omega / T$ is independent of $\beta$ and $\ell$.

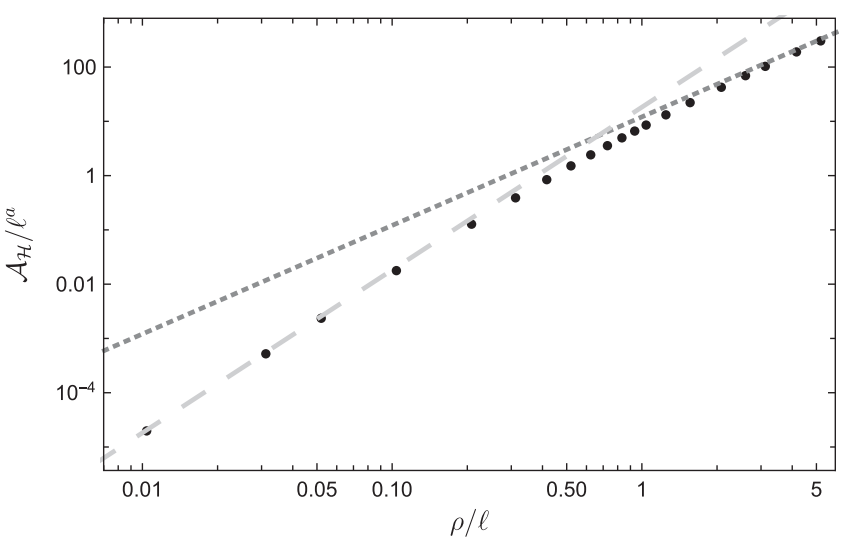

FIG. 1. The area of the bifurcating Killing surface of rotating RSII black holes, with $\alpha=0.2$, as a function of the proper radius $\rho$ (black dots) in a log-log plot. The darker, dotted line is the area of a four-dimensional Kerr black hole, while the lighter, dashed line is the area of a five-dimensional single rotating Myers Perry black hole, both of which have the same $\Omega / T$. We have divided the quantities on both axes by powers of $\ell$ to make them dimensionless, for example on the $y$ axis, we have $\mathcal{A}_{\mathcal{H}} / \ell^{a}$ where $a=3$ for both the RSII black holes and the Myers-Perry black holes, whereas $a=2$ for the Kerr black holes.

Results and discussion.-In Fig. 1 we plot the area of the bifurcating Killing sphere of rotating RSII black holes for a given value of $\alpha$ (or, equivalently, $\Omega / T$ ) against the proper radius, $\rho$ (shown as the black dots in Fig. 1). To compute $\rho$, we divide the proper distance around the equator of the braneworld black hole by $2 \pi$.

For reference, we also plot the areas of asymptotically flat five-dimensional single rotation Myers-Perry black holes (dashed, light grey line) and four-dimensional Kerr black holes (dotted, dark grey line) with the same $\Omega / T$ and appropriately scaled by powers of $\ell$, so that we only compare dimensionless quantities. For small black holes the area exhibits five-dimensional behavior, while large RSII black holes show four-dimensional behavior.

We also consider the induced geometry of the braneworld bifurcating Killing surface, i.e., where $x=1$ and $y=0$, at a constant time slice. This yields an axisymmetric two-dimensional geometry, which is completely determined by the Ricci scalar as a function of the inclination from the equatorial plane. In order to express the inclination in a gauge independent way, we introduce the function $\rho(u)$ which measures the proper radius of the circle in the two dimensional geometry at fixed $u$, so that $\rho(1)=0$ at the north pole and $\rho(u)$ increases as $u$ decreases. In Fig. 2 we plot the Ricci scalar as a function of $\rho(u)$ over $u \in(0,1)$ for each of the braneworld black holes (solid, black lines) for a fixed value of $\Omega / T$, along with the corresponding plot for the Kerr black hole (dashed, gray line). Here, and for the remainder of the paper, we multiply the quantities of both axes by the required factors of the temperature $T$ to make them dimensionless. As one moves from left to right 


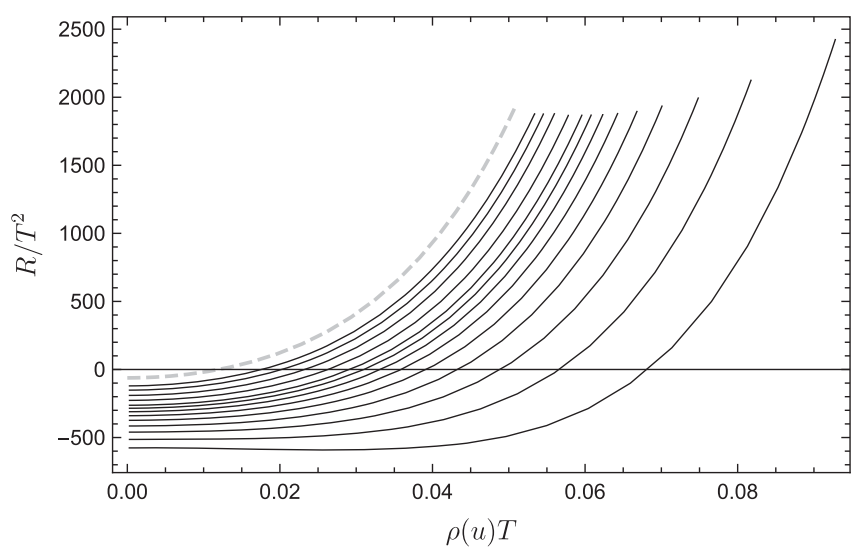

FIG. 2. The value of the Ricci scalar of the two-dimensional induced geometry of the intersection of the event horizon with the brane at constant time is plotted here for the braneworld black holes (solid, black lines) and for a four-dimensional Kerr black hole (dashed, gray line) at fixed angular velocity, $\alpha=0.6$. The braneworld curves closest to the Kerr line are those with largest $\beta$, and $\beta$ decreases monotonically as one moves between the curves from left to right.

between the different black curves in Fig. 2, $\alpha$ is fixed and $\beta$ is decreasing. The curves approach that of the Kerr black hole as $\beta$ becomes large, adding further evidence that the induced geometry of large braneworld black holes is very close to that of the Kerr geometry.

Another interesting quantity that allows for comparison to Kerr is the position of the innermost stable equatorial circular orbit (ISCO) around the black hole. We consider the motion of a massive particle restricted to the intersection of the equatorial plane $u=0$ and the brane $x=1$. Indeed, both of these planes are at the center of a $\mathbb{Z}_{2}$ symmetry in the bulk spacetime, so any geodesic starting with motion in this region will remain within it. Moreover, just like in Kerr, we have two conserved quantities associated to the two Killing vector fields: the energy $E$ and the angular momentum $h$. As usual, the use of these allows one to find an ordinary differential equation that governs the radial profile of a normalized timelike geodesic, i.e., $y(t)$, which can be written as $\dot{y}(t)^{2}=V(y ; E, h)$.

To find the ISCO, we require that $V=V^{\prime}=0$, where the derivative is with respect to $y$. These two equations can be solved to give a family of circular geodesics depending on $(E, h)$. From these, we pick the one with the minimal angular momentum, which will be the ISCO (minimizing with respect to energy gives very similar results). We computed the proper radius $\rho_{\text {ISCO }}$ of the ISCO, which once again is the proper distance around its circumference divided by $2 \pi$, and multiplied by the temperature $T$ to get a dimensionless quantity. It should be noted that this method requires some interpolation between our lattice points, so will contain some noise.

In Fig. 3, we plot the value of $\rho_{\mathrm{ISCO}} T$ against $\beta^{-1}$ for fixed $\alpha=0.5$. We expect that in the large $\beta$ limit, the

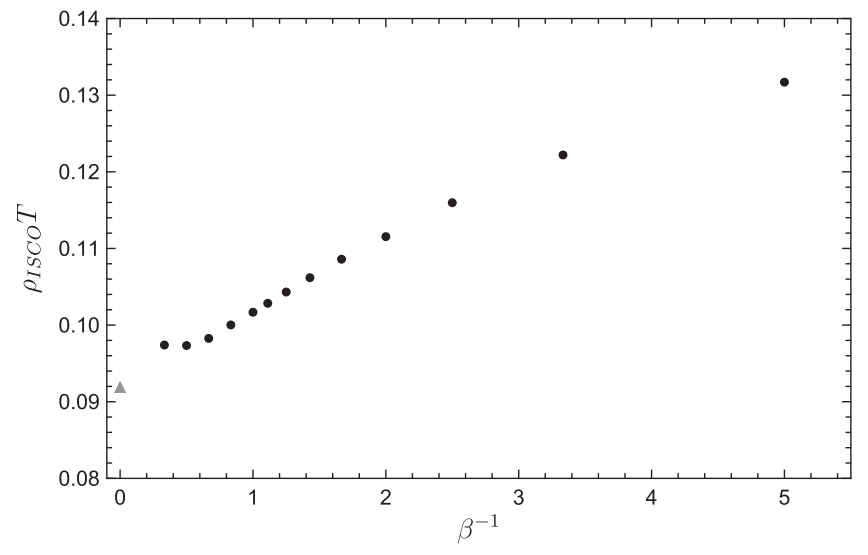

FIG. 3. The plot of the proper radius of the ISCO for braneworld black holes with $\alpha=0.5$ against $\beta^{-1}$ (black dots). We have added the corresponding value for the Kerr black hole with the same angular velocity at $\beta^{-1}=0$ (gray triangle).

braneworld black hole tends towards a Kerr geometry, thus we have added for reference the value for $\rho_{\mathrm{ISCO}} T$ for a Kerr black hole of the same angular velocity at $\beta^{-1}=0$. For each value of $\alpha$ we have probed, we observed the same qualitative behavior: $\rho_{\mathrm{ISCO}} T$ tends towards that of the Kerr black hole as $\beta$ becomes large, and increases as $\beta$ decreases. Note that, five-dimensional black holes do not contain bound orbits for timelike particles, and therefore, in agreement with our data, one would expect the value of $\rho_{\text {ISCO }} T$ to increase as $\beta$ becomes very small, where our black holes exhibit five-dimensional behavior.

We also determined the ergoregion of the RSII black holes in the bulk and found in each case that the region has spherical topology, as perhaps expected. It would be interesting to investigate whether the slightly different ergoregions for the black holes result in different superradiance instabilities $[35,36]$.

To conclude, our results clearly show a transition from five-dimensional to four-dimensional behavior as braneworld black holes increase in size, and hence that rotating braneworld black holes of finite size will differ from the standard four-dimensional Kerr black holes of general relativity. Our results discriminate in a quantitative manner the aforementioned deviations. The work presented in this Letter provides a stepping stone for imposing constraints from both multimessenger astrophysics [37] and future lepton colliders, such as the International Linear Collider $[26,27]$ and the Compact Linear Collider [28], to RandallSundrum type scenarios. Natural follow-up work includes calculating the quasi-normal mode spectrum of braneworld black holes and studying their linear mode stability. Perhaps more ambitiously, we would like to know whether the braneworld black holes we have found can be formed dynamically on the brane, following the seminal work of Ref. [38]. Finally, it would be interesting to analyze the extremal limit directly, and compare to the results reported 
in Ref. [39], which studied extremal charged black holes in RSII braneworld scenarios.

We would like to thank Harvey S. Reall and Óscar C. Dias for reading an earlier version of this Letter and for providing critical comments. J. E. S. has been partially supported by STFC consolidated Grants No. ST/P000681/1, No. ST/ T000694/1 and W. B. was supported by an STFC studentship No. ST/S505298/1 and a Vice Chancellor's award from the University of Cambridge. The numerical component of this study was carried out using the computational facilities of the Fawcett High Performance Computing system at the Faculty of Mathematics, University of Cambridge, funded by STFC consolidated Grants No. ST/P000681/1, No. ST/ T000694/1, and No. ST/P000673/1.

[1] L. Randall and R. Sundrum, An Alternative to Compactification, Phys. Rev. Lett. 83, 4690 (1999).

[2] L. Randall and R. Sundrum, A Large Mass Hierarchy from a Small Extra Dimension, Phys. Rev. Lett. 83, 3370 (1999).

[3] J. Maldacena, The large N limit of superconformal field theories and supergravity, Adv. Theor. Math. Phys. 2, 231 (1998).

[4] E. Witten, Anti-de Sitter space and holography, Adv. Theor. Math. Phys. 2, 253 (1998).

[5] O. Aharony, S. S. Gubser, J. Maldacena, H. Ooguri, and Y. Oz, Large $\mathrm{N}$ field theories, string theory and gravity, Phys. Rep. 323, 183 (2000).

[6] H. L. Verlinde, Holography and compactification, Nucl. Phys. B580, 264 (2000).

[7] S. S. Gubser, AdS/CFT and gravity, Phys. Rev. D 63, 084017 (2001).

[8] S. W. Hawking, T. Hertog, and H.S. Reall, Brane new world, Phys. Rev. D 62, 043501 (2000).

[9] R. Emparan, A. Fabbri, and N. Kaloper, Quantum black holes as holograms in AdS brane worlds, J. High Energy Phys. 08 (2002) 043.

[10] A. L. Fitzpatrick, L. Randall, and T. Wiseman, On the existence and dynamics of braneworld black holes, J. High Energy Phys. 11 (2006) 033.

[11] P. Figueras and T. Wiseman, Gravity and Large Black Holes in Randall-Sundrum II Braneworlds, Phys. Rev. Lett. 107, 081101 (2011).

[12] V.E. Hubeny, D. Marolf, and M. Rangamani, Hawking radiation in large $\mathrm{N}$ strongly-coupled field theories, Classical Quantum Gravity 27, 095015 (2010).

[13] V. E. Hubeny, D. Marolf, and M. Rangamani, Black funnels and droplets from the AdS C-metrics, Classical Quantum Gravity 27, 025001 (2010).

[14] V. E. Hubeny, D. Marolf, and M. Rangamani, Hawking radiation from AdS black holes, Classical Quantum Gravity 27, 095018 (2010).

[15] M. M. Caldarelli, O. J. C. Dias, R. Monteiro, and J. E. Santos, Black funnels and droplets in thermal equilibrium, J. High Energy Phys. 05 (2011) 116.

[16] P. Figueras, J. Lucietti, and T. Wiseman, Ricci solitons, Ricci flow, and strongly coupled CFT in the Schwarzschild
Unruh or Boulware vacua, Classical Quantum Gravity 28, 215018 (2011).

[17] J. E. Santos and B. Way, Black funnels, J. High Energy Phys. 12 (2012) 060.

[18] S. Fischetti and D. Marolf, Flowing funnels: Heat sources for field theories and the $\mathrm{AdS}_{3}$ dual of CFT 2 Hawking radiation, Classical Quantum Gravity 29, 105004 (2012).

[19] S. Fischetti, D. Marolf, and J.E. Santos, AdS flowing black funnels: Stationary AdS black holes with non-Killing horizons and heat transport in the dual CFT, Classical Quantum Gravity 30, 075001 (2013).

[20] J. E. Santos and B. Way, Black droplets, J. High Energy Phys. 08 (2014) 072.

[21] S. Fischetti, J. E. Santos, and B. Way, Dissonant black droplets and black funnels, Classical Quantum Gravity 34, 155001 (2017).

[22] D. Marolf and J. E. Santos, Phases of holographic Hawking radiation on spatially compact spacetimes, J. High Energy Phys. 10 (2019) 250.

[23] J. E. Santos, To go or not to go with the flow: Hawking radiation at strong coupling, J. High Energy Phys. 06 (2020) 104.

[24] M. Headrick, S. Kitchen, and T. Wiseman, A new approach to static numerical relativity, and its application to KaluzaKlein black holes, Classical Quantum Gravity 27, 035002 (2010).

[25] R. C. Myers and M. J. Perry, Black holes in higher dimensional space-times, Ann. Phys. (N.Y.) 172, 304 (1986).

[26] K. Fujii et al. (LCC Physics Working Group), Tests of the standard model at the international linear collider, Tech. Rep. No. (2019) [arXiv:1908.11299].

[27] H. Davoudiasl, B. Lillie, and T. G. Rizzo, Probing the universal Randall-Sundrum model at the ILC, in Proceedings of the 2005 International Linear Collider Physics and Detector Workshop and 2nd ILC Accelerator Workshop (2005) [arXiv:hep-ph/0509160].

[28] P. Roloff, U. Schnoor, R. Simoniello, and B. Xu (CLICdp Collaboration), Double Higgs boson production and Higgs self-coupling extraction at CLIC, Eur. Phys. J. C 80, 1010 (2020).

[29] See Supplemental Material at http://link.aps.org/ supplemental/10.1103/PhysRevLett.128.021601 for a description of the convergence properties of the numerical method, as well as some explicit expressions of functions and boundary conditions that are referred to in the main text, which include Ref. [30].

[30] M. Stein, Perturbative construction of stationary RandallSundrum II black holes on a 5-brane, J. High Energy Phys. 09 (2016) 067.

[31] T. Wiseman, Numerical construction of static and stationary black holes, in Black Holes in Higher Dimensions, edited by G. T. Horowitz (Cambridge University Press, Cambridge 2012).

[32] O. J. C. Dias, J. E. Santos, and B. Way, Numerical methods for finding stationary gravitational solutions, Classical Quantum Gravity 33, 133001 (2016).

[33] P. Figueras and T. Wiseman, On the existence of stationary Ricci solitons, Classical Quantum Gravity 34, 145007 (2017). 
[34] W. Israel, Singular hypersurfaces and thin shells in general relativity, Nuovo Cimento B 44S10, 1 (1966); Erratum, Nuovo Cimento B 48, 463 (1967).

[35] T. J. M. Zouros and D. M. Eardley, Instabilities of massive scalar perturbations of a rotating black hole, Ann. Phys. (N.Y.) 118, 139 (1979).

[36] S. L. Detweiler, Klein-Gordon equation and rotating black holes, Phys. Rev. D 22, 2323 (1980).
[37] P. Mészáros, D. B. Fox, C. Hanna, and K. Murase, Multimessenger astrophysics, Nat. Rev. Phys. 1, 585 (2019).

[38] D. Wang and M. W. Choptuik, Black Hole Formation in Randall-Sundrum II Braneworlds, Phys. Rev. Lett. 117, 011102 (2016).

[39] A. Kaus and H. S. Reall, Charged Randall-Sundrum black holes and $N=4$ super Yang-Mills in $\operatorname{AdS}(2) \times \mathrm{S}(2)$, J. High Energy Phys. 05 (2009) 032. 